\title{
Percepção acerca da Saúde Mental de uma equipe multiprofissional de uma emergência durante a pandemia de COVID-19
}

\author{
Perception about the Mental Health of a multidisciplinary team in an emergency during the
} COVID pandemic 19

\author{
Percepción sobre la Salud Mental de un equipo multidisciplinario en una emergencia durante la \\ pandemia de COVID-19
}

Recebido: 18/04/2021 | Revisado: 26/04/2021 | Aceito: 02/05/2021 | Publicado: 16/05/2021

\author{
Ivi Evelin Ferraz de Souza Jung \\ ORCID: https://orcid.org/0000-0002-7455-9363 \\ Universidade Federal Fluminense, Brasil \\ E-mail: ivi.jung@hotmail.com \\ Endi Evelin Ferraz Kirby \\ ORCID: https://orcid.org/0000-0002-6250-3186 \\ Instituto Nacional do Câncer, Brasil \\ Universidade Federal Fluminense, Brasil \\ E-mail: endiferraz@gmail.com \\ Ana Paula Alves Gregório \\ ORCID: https://orcid.org/0000-0001-8054-542X \\ Universidade Federal Fluminense, Brasil \\ E-mail: apag3@hotmail.com \\ Mônica Villela Gouvêa \\ ORCID: https://orcid.org/0000-0002-6552-8004 \\ Universidade Federal Fluminense, Brasil \\ E-mail: monicagouvea@gmail.com
}

\begin{abstract}
Resumo
Objetivo: Relatar a experiência acerca da percepção sobre a saúde mental de uma equipe multiprofissional de saúde durante a pandemia de COVID-19. Metodologia: Foi realizada pesquisa descritiva sobre a percepção dos profissionais de uma equipe multiprofissional de saúde, atuantes na linha de frente durante a pandemia de COVID-19, em uma Unidade de Pronto Atendimento localizada em uma cidade do estado do Rio de Janeiro. Resultados e discussão: Durante a experiência, percebeu-se a demanda de saúde mental e psicológica, além de esgotamento físico dos profissionais da unidade, como também o interesse da equipe em ter suporte de profissionais de saúde mental para o enfrentamento da pandemia de COVID-19. Considerações finais: A COVID-19 será, ainda pelos próximos anos, uma constante tanto para a comunidade científica quanto para os profissionais das equipes assistenciais de saúde. Suas alterações genéticas e sua capacidade de ter alterado a vida de todos, torna-a material de pesquisa e é de interesse geral que novos conhecimentos acerca dessa doença e suas consequências sejam compartilhados, a fim de que estejamos sempre a frente nas prevenções, cuidados e tratamentos físicos e mentais.
\end{abstract}

Palavras-chave: COVID-19; Equipe de assistência ao paciente; Emergências; Saúde mental.

\begin{abstract}
Objective: To report the experience about the perception of the mental health of a multiprofessional health team during the COVID-19 pandemic. Methodology: Descriptive research was carried out on the perception of professionals from a multiprofessional health team working on the front lines during the COVID-19 pandemic, in an Emergency Department located in a city in the state of Rio de Janeiro. Results and discussion: During the experience, the demand for mental and psychological health was perceived, in addition to the physical exhaustion of the unit's professionals, as well as the team's interest in having support from mental health professionals to face the COVID-19 pandemic. Final considerations: COVID-19 will continue to be a constant for the scientific community as well as for the professionals of health care teams for the next few years. Its genetic changes, and its capacity to have changed everyone's life, makes it research material and it is of general interest that new knowledge about this disease and its consequences be shared, so that we are always ahead in preventions, care and treatments, physical and mental.
\end{abstract}

Keywords: COVID-19; Patient assistance team; Emergencies; Mental health.

\section{Resumen}

Objetivo: Informar la experiencia sobre la percepción de la salud mental de un equipo de salud multiprofesional durante la pandemia de COVID-19. Metodología: se realizó una investigación descriptiva sobre la percepción de los 
profesionales de un equipo multiprofesional de salud que trabaja en primera línea durante la pandemia COVID-19, en una unidad de Atención de Emergencia ubicada en una ciudad del estado de Río de Janeiro. Resultados y discusión: Durante la experiencia se percibió la demanda de salud mental y psicológica, además del agotamiento físico de los profesionales de la unidad, así como el interés del equipo en contar con el apoyo de los profesionales de la salud mental para enfrentar la pandemia COVID-19. Consideraciones finales: COVID-19 seguirá siendo una constante tanto para la comunidad científica como para los profesionales de los equipos de salud durante los próximos años. Sus cambios genéticos, y su capacidad de haber cambiado la vida de todos, la convierte en material de investigación y es de interés general que se compartan nuevos conocimientos sobre esta enfermedad y sus consecuencias, de manera que estemos siempre a la vanguardia en prevenciones, cuidados y tratamientos., Físico y mental.

Palabras clave: COVID-19; Equipo de asistencia al paciente; Emergencias; Salud mental.

\section{Introdução}

A COVID-19 alcançou, por seu potencial de contaminação e morbimortalidade, o status de pandemia há cerca de um ano. Recentemente, em dezembro de 2019, houve a transmissão de um novo coronavírus (SARS-CoV-2), o qual foi identificado em Wuhan na China e causou a COVID-19, sendo em seguida disseminada e transmitida pessoa a pessoa (Brasil. Ministério da Saúde, n.d.-b), alastrando-se rapidamente para outros países ao redor do globo. Considerada inicialmente como uma doença que afetava apenas a população mais idosa, tem se mostrado, entretanto, uma afecção de todas as idades, sem predileção por grupos de risco, levando a óbito pessoas consideradas jovens e saudáveis (Bahia. Secretaria de Saúde, 2021; Goiás. Centro de Informações Estratégicas e Resposta em Vigilância em Saúde. Gerência de Vigilância Epidemiológica de Doenças Transmissíveis. Superintendência de Vigilância em Saúde, 2021).

Com números que crescem a cada dia no país e com doses de vacina que não acompanham a necessidade de vacinação de uma população com proporções continentais, a pandemia nos leva a sensação de estarmos distantes do fim. Os registros em 04 de abril de 2021, segundo o Ministério da Saúde, são de 12.953 .597 casos confirmados e 330.193 óbitos (Brasil. Secretaria de vigilância em Saúde. Ministério da Saúde, 2021). São números que assustam toda uma população, principalmente os profissionais de saúde, que têm diariamente que lidar com esses casos, em condições nem sempre favoráveis.

Quando ponderamos a pandemia e a atual situação do país ao confrontá-la, há alguns questionamentos ao pensarmos como profissionais de saúde e como equipe assistencial na linha de frente, como por exemplo: "Como me sinto com este enfrentamento?", "Sinto-me preparada para esta situação?", “O que posso fazer para melhorar minha saúde mental em tempos de pandemia?".

Não é de hoje que os profissionais de saúde lidam com circunstâncias que podem desencadear estresse emocional. Lidar com a dor do outro é algo que pode trazer, através da empatia, certo sofrimento, dependendo das situações a que este profissional estiver exposto. Somando-se a isto toda a carga emocional, como talvez a sensação de culpa por ter que se expor, expondo também seus familiares, podendo causar um isolamento ainda maior e a estigmatização (Buselli, Baldanzi, Corsi, Chiumiento, Del Lupo, Carmassi, et al., 2020).

As equipes de unidades de pronto atendimento são equipes dinâmicas, que atuam em diversas situações que caracterizam risco iminente de morte ao paciente, sendo equipes que estão em constante contato com desfecho óbito podendo trazer ainda mais ansiedade àquele profissional atuante. As Unidades de Pronto Atendimento fazem parte da Rede de Atenção às Urgências e são unidades intermediárias e que por serem portas abertas, na maioria das vezes, são locais onde ocorre o primeiro atendimento ao paciente, onde ele é estabilizado para posteriormente dar continuidade ao tratamento (Brasil. Ministério da Saúde, s.d.).

Importante seria que instituições e gestores se preocupassem não tão somente com protocolos, equipamentos de proteção individual e todo o gerenciamento funcional da pandemia de COVID-19, como também dessem suporte psicológico e emocional aos seus profissionais. Segundo a Organização Mundial da Saúde (OMS), o primeiro passo para proteger a saúde 
dos profissionais em uma crise epidêmica é a implementação de todas as medidas necessárias para proteger a sua segurança ocupacional, o que inclui a sua saúde mental (Buselli, Baldanzi, Corsi, Chiumiento, Del Lupo, Carmassi, et al., 2020).

Neste contexto, o estudo tem como objetivo descrever a percepção sobre a saúde mental de uma equipe multiprofissional de saúde durante a pandemia de COVID-19 e descrever possíveis intervenções para a melhoria da saúde mental dos profissionais, com base na literatura disponível.

\section{Metodologia}

Foi realizada pesquisa descritiva sobre a percepção dos profissionais de uma equipe multiprofissional de saúde, atuantes na linha de frente durante a pandemia de COVID-19. O presente estudo foi realizado com base na observação e conversas informais com profissionais de uma equipe multiprofissional de saúde, atuantes em uma unidade de pronto atendimento de uma cidade do Rio de Janeiro, tendo como motivação a percepção sobre o assunto em questão, que ocorreu no início da pandemia de COVID-19, progredindo com o passar do tempo para o aumento gradual do número de casos, levando há um progressivo aumento de trabalho para a equipe, ocasionando aumento de demanda física e emocional.

Foram feitas pesquisas bibliográficas em sites do Ministério da Saúde, governos de estados e na plataforma Biblioteca Virtual em Saúde.

\section{Resultados e Discussão}

Ao ouvirmos as primeiras notícias acerca de um vírus recém-descrito na cidade de Wuhan na China, não imaginávamos que demoraria tão pouco para desencadear uma pandemia e tampouco que essa realidade chegaria tão rápido em nosso cotidiano de trabalho e em nossa vida social. Uma vez a transmissão pessoa a pessoa instalada no país, foram tomadas diversas medidas para tentar a contenção dessa transmissão, entre elas o distanciamento social, a interrupção das aulas e o fechamento dos comércios, dividindo muitas opiniões e causando também muita ansiedade (Lopes \& Cordeiro, 2021).

Há, ainda hoje, após um ano de seu curso, a sensação latente de impotência diante de um vírus que tem dominado a todos de tal forma a nos deixar exaustos do trabalho, do cuidado com os outros e para conosco, da preocupação com os colegas de trabalho e com os familiares.

De fato, tem sido jornadas de trabalho pesadas, colegas afastados e outros tantos desempenhando jornadas maiores, a fim de fazer cumprir o cronograma de trabalho da unidade, além da constante frustração com óbitos de pacientes a quem devotam cuidados. A sensação de não valorização profissional pode aumentar a tristeza e os medos, traduzindo-se inclusive em sua vida pessoal (Kirby, Siqueira, Cunha, Santiago, Neves, \& Beserra 2021).

Situações novas, com potencial para causar danos, como é próprio das emergências, situações que causam ansiedade, podem trazer ao profissional que lida com este cotidiano algumas alterações emocionais, sintomas de algum distúrbio de ansiedade, depressão, por exemplo, que podem ser ou não passageiros (Chapa-Koloffon, Jean-Tron, Ávila-Hernández, Márquez-González, \& Garduño-Espinosa, 2021; Menon, Narayan, \& Bhade, 2021).

A acumulação de alguns fatores anteriores a pandemia traz, por si só, o desafio da atuação na área da saúde, equipes reduzidas, salários baixos, insegurança com os riscos próprios da profissão, somados ao que se sabe sobre o SARS-cov-2 como alta transmissibilidade e a alta mortalidade que temos acompanhado, a escassez de equipamentos com aumento gradativo da demanda e as mudanças nas escalas de trabalho aumentam as chances de esgotamento tanto físico quanto emocional (Buselli, Baldanzi, Corsi, Chiumiento, Del Lupo, Carmassi, et al., 2020; Buselli, Corsi, Veltri, Baldanzi, Chiumiento, Lupo, Del, et al., 2021).

Em algumas unidades de saúde e nesta unidade em questão foram montadas tendas de atendimento à demanda de COVID-19, a fim de organizar o fluxo e separar os atendimentos dos pacientes não suspeitos de covid durante os horários de 
maior fluxo.

Em conversa com alguns dos profissionais atuantes na tenda, o que ficou demonstrado em suas falas foi que a equipe está sobrecarregada, com sensação de esgotamento físico e mental, com carga de trabalho aumentando a cada dia e a quantidade de pacientes mais graves também. Essa é a percepção de muitos colegas: o agravamento dos casos, os pacientes dando entrada nas salas vermelha e amarela, a saturação baixa, o risco de evoluir para óbito, além do aumento no número de casos suspeitos, aumento nas testagens de suspeitos respiratórios e aumento na quantidade de notificações compulsórias a serem preenchidas.

Foi possível observar que os profissionais da odontologia foram os primeiros a receber informações da categoria para interromper os atendimentos não emergenciais, enquanto não tivessem protocolos com redução de riscos para a classe. A forma de contágio do novo coronavírus se dá por contato direto, saliva, gotículas e aerossóis e, o atendimento odontológico produz esses fatores, sendo então realmente necessário o estudo e o estabelecimento de maneiras de prevenção da saúde destes trabalhadores, não sendo, por este motivo, infundadas as preocupações com os atendimentos dessa classe profissional (Quincho-Rosales, D. A., Castro-Rodríguez, Y., \& Grados-Pomarino, S., 2020).

Muitos protocolos foram instituídos, para todas as categorias, desde o início da pandemia até o presente momento e, esse também passa a ser um agente estressor para alguns profissionais, visto que tudo muda muito rápido, sendo por vezes difícil acompanhar. Mensagens conflitantes das autoridades, como mudanças nos protocolos e mudanças nos usos de equipamentos de proteção individual, aliados a quarentena prolongada, a imprevisibilidade da doença e a perda de liberdades são fatores que podem desencadear estresse (Bueno-Notivol, Gracia-García, Olaya, Lasheras, López-Antón, \& Santabárbara, 2021).

Toda uma estrutura que antes funcionava sem a pandemia vem se reestruturando para enfrentá-la, envolvendo gestores, administração e outros profissionais, que mesmo não sendo assistenciais, não estão menos envolvidos com as mudanças trazidas com essa nova situação. O sentimento é compartilhado, cobranças que aumentaram, demanda de trabalho crescente e esgotamento físico e emocional. As unidades de saúde tiveram que se reestruturar, alterar fluxos de atendimentos, separando os suspeitos respiratórios de pacientes com afecções diversas (Santiago \& Da Silva, 2020).

Alguns descreveram sentimentos de paranoia e medo, medo de se contaminarem ou a alguém da família e a amigos, medo do que está por vir, das mudanças, do enfrentamento de óbitos e de não enxergar um fim tão próximo da pandemia. Esses anseios se dão, principalmente, quando ponderamos a questão das novas mutações genéticas do vírus, sabemos que, em se tratando desses micro-organismos, mutações são condições esperadas, porém, para o profissional pode trazer ainda mais a ansiedade do desconhecido. Apesar de ainda não terem sido descritas na literatura que estas mutações sejam mais graves, estudos demonstram serem ainda mais transmissíveis (Fundação Oswaldo Cruz, 2021).

Outra variante a ser considerada e que foi descrita pelos profissionais é a possibilidade de uma reinfecção. Muitos dos profissionais da unidade foram testados para COVID-19 com resultado positivo, o que por algum tempo trouxe a alguns a sensação de uma proteção adquirida, porém estudos vêm demonstrando casos de reinfecção, com possibilidades tanto por cepas diferentes quanto por uma mesma cepa (Brasil. Coordenação-Geral do Programa Nacional de Imunizações. Departamento de Imunização e Doenças Transmissíveis. Secretaria de Vigilância em Saúde. Ministério da Saúde, 2020).

Os profissionais demonstraram o desejo de terem acompanhamento psicológico, algum tipo de terapia, um tratamento que os auxiliassem no enfrentamento a todas essas condições que surgiram com a pandemia de COVID-19. Alguns estudos discorrem sobre a necessidade do cuidado com a saúde mental da equipe profissional, principalmente durante a pandemia, com a atuação de profissionais psiquiatras, psicólogos, rede de atenção online com profissionais preparados ou mesmo com auxílio espiritual e religioso com suporte de capelania, por exemplo, posto que o suporte é baseado na estrutura psicossocial dos indivíduos (Buselli, Baldanzi, Corsi, Chiumiento, Del Lupo, Carmassi, et al., 2020.; Rajkumar, 2020). 
Recente estudo demonstrou ainda os efeitos das terapias alternativas em saúde ao utilizar-se da auriculoterapia para auxiliar a baixar os níveis de estresse, ansiedade e depressão apresentados pelos profissionais participantes do estudo, durante a pandemia de COVID-19. A técnica chinesa consiste em pressionar pontos energéticos, onde no estudo foram utilizadas sementes de mostarda que pressionavam determinado ponto por aproximadamente cinco minutos (Oliveira, Assis, Mendes, Lemos, Sousa, \& Chianca, 2021).

A criação de um ambiente de trabalho aberto ao diálogo, com interação entre os profissionais, destacando a comunicação entre as equipes, ouvindo medos e incertezas, cria oportunidade para que os profissionais possam expressar seus sentimentos, fazendo com que se sintam ouvidos e que suas emoções tenham espaço e sejam validadas (Kirby, Siqueira, Cunha, Santiago, Neves, \& Beserra, 2021).

O que nos permite prever com o avanço da pandemia e o número de óbitos, que hoje já é registrado no país, com todas as mudanças e estressores a que os profissionais foram submetidos é que será primordial que sejam oferecidos, se não agora, em um futuro próximo, tratamentos que visem dar um suporte de saúde mental aos profissionais (Best, Rajaee, \& Vandenhoeck, 2021).

Com a oferta de um tratamento, teria o profissional a chance de procurar este apoio, caso sentisse necessidade, diminuindo com isso os riscos futuros de afastamento do serviço por questões psicológicas e de saúde mental, constituindo essa oferta de tratamento como uma prevenção também a eventuais afastamentos de profissionais do serviço, por danos à saúde mental, em um momento em que muitos já são afastados por contrair a COVID-19.

O estudo apresentou limitações por não haver contemplado todos os profissionais do quadro efetivo da unidade, não tendo sido possível ouvir todas as categorias profissionais atuantes na unidade.

É uma investigação qualitativa e teve como base a percepção das autoras acerca de relatos e conversas informais com membros da equipe multiprofissional de saúde.

\section{Considerações Finais}

Muitos são os desafios para profissionais de saúde durante a pandemia de COVID-19, com jornadas de trabalho aumentadas, déficits de equipamentos e colapso no Sistema de Saúde Pública, aliado há outras variantes como medos, inseguranças, esgotamento físico e mental desencadeando problemas psíquicos e emocionais.

A COVID-19 será ainda pelos próximos anos uma constante, tanto para a comunidade científica quanto para os profissionais das equipes assistenciais de saúde. Suas alterações genéticas e sua capacidade de ter alterado a vida de todos, torna-a material de pesquisa e é de interesse geral que novos conhecimentos acerca dessa doença e suas consequências sejam compartilhados, a fim de que estejamos sempre a frente nas prevenções, cuidados e tratamentos físicos e mentais. Por se tratar de um tema atual e relevante, sugerimos que estudos futuros sejam desenvolvidos, abrangendo diversas categorias profissionais e em diferentes cenários da saúde, a fim de ampliar os conhecimentos nesta área.

\section{Referências}

Bahia. Secretaria de Saúde. (2021). Notícias: Mortes entre jovens com COVID-19 crescem 447\% na Bahia. http://www.saude.ba.gov.br/2021/03/30/mortesentre-jovens-com-covid-19-crescem-447-na-bahia/

Best, M., Rajaee, G., \& Vandenhoeck, A. (2021). A Long Way to Go Understanding the Role of Chaplaincy? A Critical Reflection on the Findings of the Survey Examining Chaplaincy Responses to Covid-19. Journal of Pastoral Care \& Counseling: Advancing Theory and Professional Practice through Scholarly and Reflective Publications, 75(1), 46-48. https://doi.org/10.1177/1542305021992002

Brasil. Coordenação-Geral do Programa Nacional de Imunizações. Departamento de Imunização e Doenças Transmissíveis. Secretaria de Vigilância em Saúde. Ministério da Saúde. (2020). Nota técnica no 52/2020 - CGPNI/DEIDT/SVS/MS (52/2020). https://www.gov.br/saude/ptbr/media/pdf/2020/dezembro/10/11-sei_nota-reinfeccao.pdf 
Brasil. Ministério da Saúde. (s.d.). Unidade de Pronto Atendimento (UPA 24h): o que é, quando usar, diretrizes e competências. https://antigo.saude.gov.br/saude-de-a-z/unidade-de-pronto-atendimento-upa-24h.

Brasil. Ministério da Saúde. (n.d.-b). Sobre a doença. https://coronavirus.saude.gov.br/sobre-a-doenca\#o-que-e-covid

Brasil. Secretaria de vigilância em Saúde. Ministério da Saúde. (2021). Painel Coronavírus. https://covid.saude.gov.br/

Bueno-Notivol, J., Gracia-García, P., Olaya, B., Lasheras, I., López-Antón, R., \& Santabárbara, J. (2021). Prevalence of depression during the COVID-19 outbreak: A meta-analysis of community-based studies. International Journal of Clinical and Health Psychology, 21(1). https://doi.org/10.1016/j.ijchp.2020.07.007

Buselli, R., Baldanzi, S., Corsi, M., Chiumiento, M., Del Lupo, E., Carmassi, C., Dell'Osso, L., \& Cristaudo, A. (2020). Psychological care of healthworkers during the COVID-19 outbreak in Italy: Preliminary report of an occupational health department (AOUP) responsible for monitoring hospital staff condition. Sustainability (Switzerland), 12(12), 1-16. https://doi.org/10.3390/su12125039

Buselli, R., Corsi, M., Veltri, A., Baldanzi, S., Chiumiento, M., Lupo, E. Del, Marino, R., Necciari, G., Caldi, F., Foddis, R., Guglielmi, G., \& Cristaudo, A. (2021). Mental health of Health Care Workers (HCWs): a review of organizational interventions put in place by local institutions to cope with new psychosocial challenges resulting from COVID-19. Psychiatry Research, 299 113847. https://doi.org/10.1016/j.psychres.2021.113847

Chapa-Koloffon, G. D. C., Jean-Tron, M. G., Ávila-Hernández, A. V., Márquez-González, H., \& Garduño-Espinosa, J. (2021). Frequency of acute stress disorder in health care workers of a tertiary level pediatric hospital during the national safe distance strategy for covid-19 prevention. Boletin Medico Del Hospital Infantil de Mexico, 78(1), 10-17. https://doi.org/10.24875/BMHIM.20000226

Fundação Oswaldo Cruz. (2021). Rede Genômica Fiocruz. http://www.genomahcov.fiocruz.br/

Goiás. Centro de Informações Estratégicas e Resposta em Vigilância em Saúde. Gerência de Vigilância Epidemiológica de Doenças Transmissíveis. Superintendência de Vigilância em Saúde. (2021). Boletim Epidemiológico COVID-19 no44 - 05/02/2021 SITUAÇÃO EPIDEMIOLÓGICA (04/02/2020 a 30/01/2021) (907, 44). https://www.saude.go.gov.br/files/boletins/epidemiologicos/boletim44.pdf

Kirby, E. E. F., Siqueira, A. S. de A., Cunha, D. A. de O. da, Santiago, F. B., Neves, L. M. L., \& Beserra, V. dos S. (2021). Covid-19 And Its Psychical Influences On The Perception Of The Nursing Staff Of Oncological Palliative Care. Rev Min Enferm, 25, e-1355. https://doi.org/10.5935/1415-276220210003

Lopes, M. B., \& Cordeiro, B. C. (2021). Estratégia de ensino durante a pandemia de COVID-19 para profissionais da vigilância em saúde, Minas Gerais: um relato de experiência Teaching strategy during the COVID-19 pandemic for health surveillance professionals, Minas Gerais : an experience rep. 2021, 1-6.

Martínez Menchaca, H. R., González Solís, A., \& Juárez Casanova, K. (2020). Procedimientos libres de aerosol ante COVID-19 en odontología pediátrica. Revista de La Asociación Dental Mexicana, 77(6), 301-305. https://doi.org/10.35366/97618

Menon, B., Narayan, S. K., \& Bhade, S. (2021). COVID-19, Moral Injury and the Bhagvad Gita. Journal of Religion and Health, 60(2), 654-662. https://doi.org/10.1007/s10943-021-01210-z

Oliveira, C. M. C. de, Assis, B. B. de, Mendes, P. G., Lemos, I. C., Sousa, A. L. C. de, \& Chianca, T. C. M. (2021). Auriculoterapia em profissionais de enfermagem na pandemia do coronavírus: estudo de casos múltiplos. Revista Eletrônica de Enfermagem, 23, 1-9. https://doi.org/10.5216/ree.v23.65678

Quincho-Rosales, D. A., Castro-Rodríguez, Y., \& Grados-Pomarino, S. (2020). Consideraciones sobre la atención estomatológica en el Perú durante la pandemia por la COVID-19. In Revista Cubana de Estomatología (57, e3315). scielocu. http://scielo.sld.cu/scielo.php?script=sci_arttext\&pid=S0034$75072020000300005 \& \operatorname{lng}=$ es\&nrm=iso

Rajkumar, R. P. (2020). Since January 2020 Elsevier has created a COVID-19 resource centre with free information in English and Mandarin on the novel coronavirus COVID- 19. The COVID-19 resource centre is hosted on Elsevier Connect, the company's public news and information. Asian Journal of Psychiatry, 52(March), 1-5.

Santiago, F. B., \& Da Silva, A. L. A. (2020). Primeiro caso de COVID-19 em uma unidade de Cuidados Paliativos oncológicos: relato de experiência. Enfermagem Em Foco, 11(2.ESP), 205-210. https://doi.org/10.21675/2357-707x.2020.v11.n2.esp.3847 